\title{
Microwave Absorption Properties of Garnet Ferrite Filled Polymer Nanocomposites in X-Band
}

\author{
S.M.Alami , H.Ammor \\ souhail.alami.m@gmail.com, ammor@emi.ac.ma \\ Engineering 3S Research Center,Smart Communications Research Team \\ Mohammadia School of Engineering, Mohammed V University in Rabat-Morocco
}

\begin{abstract}
The electromagnetic properties of ferrite filled polymer nano-composites can be determined by a classical Nicolson-Ross and Weir technique. The measurement of Sparameters makes it possible to calculate the complex permittivity $\varepsilon$ and permeability $\mu$ of the Lanthanum Iron Garnet (LIG)-loaded polyvinylidene fluoride (PVDF )-polymer nanocomposites over the $\mathrm{X}$-band waveguide. The reflection $S_{11}$ and transmission $S_{21}$ coefficients were calculated by the transmission/reflection rectangular waveguide technique. The LIG/PVDF sample, placed in the rectangular waveguide over X-band frequencies. The average values of the relative complex permittivity and permeability of LIG/PVDF over X-band frequencies [8 - $12 \mathrm{GHz}]$ are $(4.34-\mathrm{j} 0.09)$ and $(1.27-\mathrm{j} 0.15)$ respectively.
\end{abstract}

Keywords: Complex permittivity and permeability, Ferrite, Garnet, LIG, PVDF, waveguide W-90, X-band.

\section{Introduction}

Ferrites are ferrimagnetic oxides materials have been heavily used in passive microwave devices such as circulators, isolators, phase shifters, resonators, filters, , and miniature antennas [1]. The garnets are soft ferrites having the general chemical formula $\mathrm{R}_{3} \mathrm{Fe}_{5} \mathrm{O}_{12}$ where $\mathrm{R}$ is yttrium or trivalent rare earth ions from lanthanum to ytterbium[2]. Garnet ferrites have a cubic crystal structure and include three types of crystallographic lattice of (a,b and c), the $3 \mathrm{R}^{3+}$ ions are located the dodecahedra (c) site, $2 \mathrm{Fe}^{2+}$ ions are distributed to the octahedral (a) site and $3 \mathrm{Fe}^{3+}$ ions occuping the tetrahedral (d) site, where as $\mathrm{O}^{2-}$ ions are located to the interstitial sites [3].

Lanthanum Iron Garnet (LIG) has a chemical formula $\mathrm{La}_{3} \mathrm{Fe}_{5} \mathrm{O}_{12}$, it is strongly used in electronic devices, due to the efficient absorption of electromagnetic waves, low permeability, low losses at high frequencies, extremely easy to magnetize and demagnetize, low saturation flux, and high resistivity. In recent years, many works have been made on polymer matrix based nanocomposites filled with ferrite particles, such as Co-ferrite [4], Ni-Zn-ferrite [5], and $\mathrm{Mn}-\mathrm{Zn}$-ferrite [6], using different characterization methods [7].

In this paper, the transmission/reflection (TR) caracterization method based on NicolsonRoss-Weir approach allows to calculate simultaneously the complex relative permittivity and complex relative permeability of the lanthanum iron garnet filled -PVDF polymer nanocomposite, from the S-parameters, the sample was placed in a rectangular waveguide over the X-band frequencies [8-12 GHz], and with the ANSYS HFSS simulation, we can 
measure the reflection and transmission coefficients and calculate the complex relative permittivity and complex relative permeability of LIG/PVDF composites.

\section{Materials and methods}

Many papers for the physico-chemical synthesis of lanthanum iron garnet have been described in $[8,9]$ by sol-gel procedure.

\subsection{Preparation of the LIG powder}

The procedure for preparing LIG magnetic powders is shown in the diagram below. Figure 1.

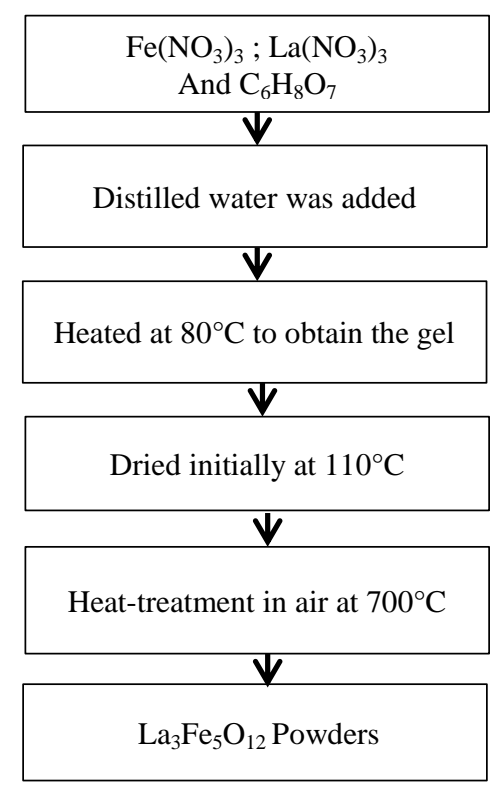

Fig. 1. Procedure for preparing Lanthanum Iiron Garnet powders

The LIG/PVDF as a nanocomposite was prepared by the solvent method, with $13 \%$ LIG nano-powders and $87 \%$ of PVDF plymer in the shape of a rectangular sheet $3 \mathrm{~mm}$ thickness. 


\subsection{Simulation of the rectangular waveguide loaded with the sample LIG/PVDF}

The PVDF-LIG nano-composite sample of dimensions $\left(22.86 * 11.43 * 3 \mathrm{~mm}^{3}\right)$, was well adjusted into a rectangular waveguide cross section WR-90. The S-parameters then were measured in the frequency range from 8 to $12 \mathrm{GHz}$ by using the HFSS simulator. The simulation can be shown in Figure 2.

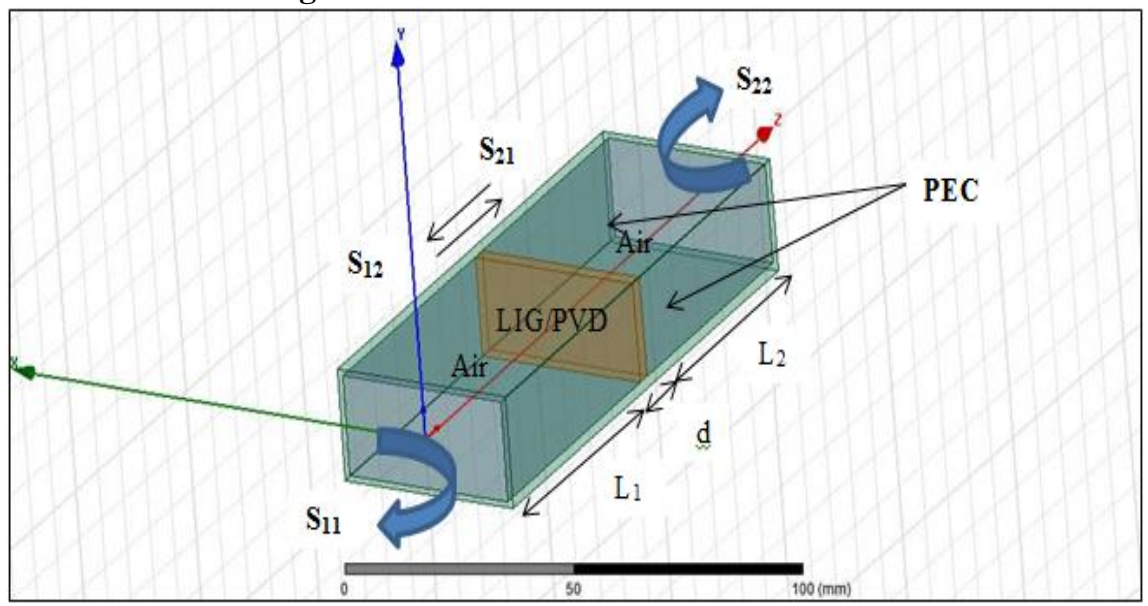

Fig. 2. Simulation of the rectangular waveguide

\subsection{Nicholson-Ross-Weir (NRW) method}

Determination of the electromagnetic properties of PVDF-LIG composite sample using transmission/reflection rectangular waveguide technique[10-12]. The organigram shown in the Figure 3 describes the procedure for extracting the S-parameters and estimation complex permeability and permittivity of the sample under study.

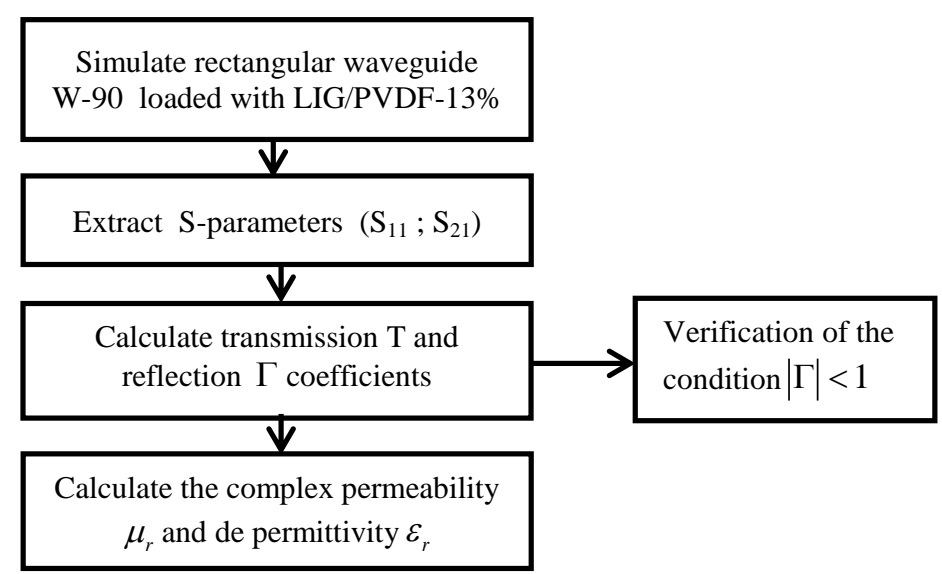

Fig. 3. Algorithm of Nicolson-Ross and Weir 
The proposed classical procedure by Nicolson-Ross and Weir is based on the following formulas, these equations are applicable for the rectangular waveguide with the transverse electric $\mathrm{TE}_{10}$ mode.

$$
\begin{aligned}
& S_{11}=R_{1}^{2} \frac{\Gamma\left(1-T^{2}\right)}{1-\Gamma^{2} T^{2}} \\
& S_{21}=R_{1} \times R_{2} \frac{T\left(1-\Gamma^{2}\right)}{1-\Gamma^{2} T^{2}} \\
& R_{1}=\exp \left(-\gamma_{0} L_{1}\right) \text { and } R_{2}=\exp \left(-\gamma_{0} L_{2}\right) \\
& T=\exp (-\gamma d) \\
& \gamma=j \frac{2 \pi}{\lambda_{0}} \sqrt{\varepsilon_{r} \mu_{r}-\left(\frac{\lambda_{0}}{\lambda_{C}}\right)^{2}} \\
& \gamma_{0}=j \frac{2 \pi}{\lambda_{0}} \sqrt{1-\left(\frac{\lambda_{0}}{\lambda_{C}}\right)^{2}} \\
& \Gamma=K \pm \sqrt{K^{2}-1} \\
& K=\frac{S_{11}^{2}-S_{21}^{2}+1}{2 S_{11}} \\
& T=\frac{S_{11}+S_{21}-\Gamma}{1-\left(S_{11}+S_{21}\right) \Gamma} \\
& \mu_{r}=j\left(\frac{1+\Gamma}{1-\Gamma}\right) \times \frac{c}{2 \pi f d} \ln (T) \\
& \varepsilon_{r}=-\left(\frac{c}{2 \pi f d} \ln (T)\right)^{2} \times \frac{1}{\mu_{r}}
\end{aligned}
$$


- $\Gamma$ and $\mathrm{T}$ are respectively the reflection and transmission coefficients;

$-\gamma_{0}$ and $\gamma$ are respectively the propagation constants in the vacuum and sample;

$-\lambda_{0}$ and $\lambda_{C}$ represent respectively the free space and the cut-off wavelengths;

$-\varepsilon_{r}$ et $\mu_{r}$ are the relative permittivity and permeability respectively.

$-\mathrm{R}_{1}$ and $\mathrm{R}_{2}$ correspond the expression of reference planes respectively.

$-\mathrm{L}_{1}$ and $\mathrm{L}_{2}$ are the respective distances from calibration reference planes to sample ends.

$-d$ is wavelenght of sample.

\section{Results and discussion}

The Nicolson-Ross and Weir (NRW) technique was used to calculate the complex permittivity and permeability of LIG/PVDF nanocomposite, from reflection $S_{11}$ and transmission $S_{21}$ coefficients using the transmission/reflection (TR) measurement technique. Figure 4a. below shows two response the curves of LIG/PVDF sample of the real permittivity and imaginary permittivity as function of frequency. The real part of permittivity is decreasing from $8 \mathrm{GHz}$ to $12 \mathrm{GHz}$, this real part has a high value nearly 6.08 in frequency at $8 \mathrm{GHz}$, and then decreases to 3.45 at $12 \mathrm{GHz}$ when the frequency increases. This figure also represents the imaginary part of the permittivity curve. The imaginary part has a small variation with frequency increases from $8 \mathrm{GHz}$ to $12 \mathrm{GHz}$. The observed response of the permittivity constant is the result of adding the portion of the LIG magnetic in PVDF polymer matrix [13].

The Figure 4b. below, presents two response the curves of LIG/PVDF nanocomposite of the real permeability and imaginary permeability as function of frequency. it illustrates that the real part of permeability is 3.53 at $8 \mathrm{GHz}$, from this point, the curve of the real permeability shows a gradual decrease with increasing frequency to reach 0.23 at $12 \mathrm{GHz}$.

Similarly, the imaginary part decreases from 0.75 to 0.01 with increasing frequency from $8 \mathrm{GHz}$ to $12 \mathrm{GHz}$, the decreasing is the product of the domain wall motion and the spine-lattice interaction.

The average values obtained in this work of the complex permittivity and permeability respectively of mentioned above nanocomposite in X-band frequencies were (4.34-j0.09) and (1.27-j0.15). The results achieved in this project are in excellent agreement with the literature[14]. 

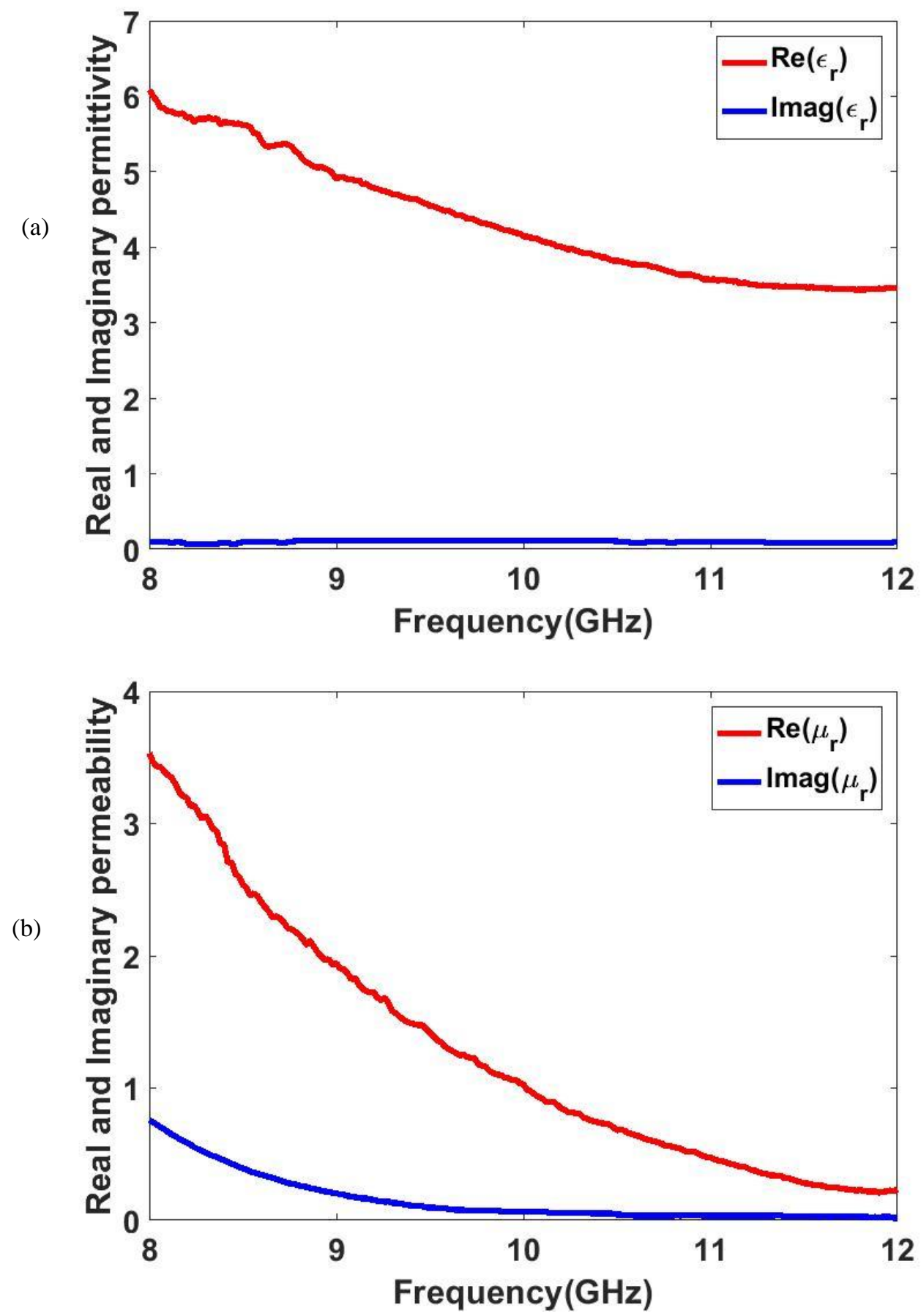

Fig. 4. Evolution of (a) Complex permittivity and (b) Complex permeability of LIG/PVDF nanocomposite versus frequency 
Table 1. below represents the comparison between the values calculated and measured [14].

Table 1. Comparison table between the calculated parameters and the measured parameters

\begin{tabular}{|l|c|c|c|c|}
\hline LIG/PVDF-13\% & $\varepsilon_{r}$ & $\mu_{r}$ & Error $\frac{\Delta \varepsilon_{r}^{\prime}}{\varepsilon_{r}^{\prime}}$ & Error $\frac{\Delta \mu_{r}^{\prime}}{\mu_{r}^{\prime}}$ \\
\hline Calculed & $4.34-\mathrm{j} 0.09$ & $1.27-\mathrm{j} 0.15$ & $\leq 0.23 \%$ & $\leq 2.3 \%$ \\
\cline { 1 - 3 } Measured & $4.33-\mathrm{j} 0.09$ & $1.24-\mathrm{j} 0.15$ & & \\
\hline
\end{tabular}

\section{Conclusions and perspectives}

In this paper, the LIG/PVDF nanocomposite has been fabricated in the shape of rectangular sheets with thickness of $3 \mathrm{~mm}$. The S-parameters were measured by the cross section rectangular waveguide at the X-band. The Nicholson-Ross-Weir (NRW) procedure was employed to calculate the complex permittivity and complex permeability of the LIG/PVDF sample. The results indicated at X-band frequencies, show the both real part of permittivity, real part of permeability and imaginary part of complex permeability are decreased when increasing the frequency in X-band. But, the imaginary part of the dielectric permittivity probably remains constant in this frequency range. The average values obtained in this work of the complex permittivity and permeability respectively of mentioned above nanocomposite in X-band frequencies were (4.34-j0.09) and (1.27-j0.15). The results achieved in this project are in excellent agreement with the literature[14].

\section{Acknowledgement}

The authors would like to thank the members of Institute of Electronics and Telecommunications of Rennes, France for allowing us to use the commercial solvers available in their laboratory.

\section{References}

[1] Y.J. Wu, H.P. Fu, R.Y. Hong, Y. Zheng, D.G. Wei.: Influence of surfactants on co-precipitation synthesis of Bi-YIG particles. Vol. 470, $\mathrm{N}^{\circ} 1-2$, pp. 497-501. Journal of Alloys and Compounds (2009) [2] Ümit Özgüri, Yahya Alivov, and Hadis Morkoç.: Microwave Ferrites, Part 1: Fundamental properties. Vol. 20, N9, pp. 789-834. Journal of Materials Science: Materials in Electronics (2009) 
[3] Makiyyu Abdullahi Musa, Raba'ah Syahidah Azis, Nurul Huda Osman, Jumiah Hassan, Tasiu Zangina.: Structural and magnetic properties of yttrium iron garnet (YIG) and yttrium aluminum iron garnet (YAlG) nanoferrite via sol-gel synthesis. Vol. 7, pp. 1135-1142. Results in Physics (2017)

[4] Vaishnava PP, Senaratne U, Buc E, Naik R, Naik VM, Tsoi G, et al.: Magnetic properties of cobaltferrite nanoparticles embedded in polystyrene resin. Vol. 99, $\mathrm{N}^{\circ}$ 08G702, pp. 1-3. J. Appl Phys (2006)

[5] Ting, T. H.; Yu, R. P.; Jau, Y. N.: Synthesis and Microwave Absorption Characteristics of Polyaniline/NiZn Ferrite Composites in 2-40 GHz. Vol. 126, pp. 364-368. Mater. Chem. Phys (2011)

[6] Amiri, Gh. R.; Yousefi, M. H.; Abolhassani, M. R.; Manouchehri, S.; Keshavarz, M. H.; Fatahian, S. : Magnetic Properties and Microwave Absorption in Ni-Zn and Mn-Zn Ferrite Nanoparticles Synthesized by Low-Temperature Solid-State Reaction. Vol. 323, №6 pp. 730-734. J. Magn. Magn. Mater (2011)

[7] H. Elmajid, J. Terhzaz, H. Ammor.: Implementation of the Mode Matching Technique to Estimate the Complex Permittivity of Dielectric Material at Microwave Frequency Using Rectangular Waveguide Measurements. Vo1. 108, pp. 123-138. European Journal of Scientific Research ( 2013)

[8] P. Vaqueiro, M. P. Crosnier-Lopez, and M. A. Lopez-Quintela.: Synthesis and Characterization of Yttrium Iron Garnet Nanoparticles. Vol. 126, $\mathrm{N}^{\circ} 0324$ pp. 161-168. Journal of solid state chemistry (1996)

[9] Majid Niaz Akhtar, Noorhana Yahya, and Nadeem Nasir.: New EM Transmitter with Y3Fe5O12 based Magnetic Feeders Potentially Used for Seabed Logging Application. Vol. 667, pp 10-23. Advanced Materials Research ( 2013)

[10] A. M. Nicolson and G. F. Ross.: Measurement of the Intrinsic Properties of Materials by Timedomain Techniques. Vol. 19, Nº , pp. 377-383. IEEE Trans. Instrum. Meas (1970)

[11] A. M. Nicolson.: Broad-band Microwave Transmission Characteristics From a Single Measurement of the Transient Response. Vol. 17, N4, pp. 395402. IEEE Trans.Instrum. Meas (1968)

[12] W. B. Weir.: Automatic Measurements of Complex Dielectric Constant and Permeability at Microwave Frequencies. Vol. 62, N¹, pp. 3339. Proc.IEEE (1974)

[13] Christian Brosseau, Jamal Ben Youssef, Philippe Talbot, and Anne-Marie Konn.: Electromagnetic and magnetic properties of multicomponent metal oxides heterostructures: Nanometer versus micrometer-sized particles. Vol. 93, N 11, 1, pp. 9243- 9256. J. Appl. Phys (2003)

[14] Hassan Soleimani, Zulkifly Abbas, Noorhana Yahya, Hojjatollah Soleimani,Mohammad Yeganeh Ghotbi.: Determination of complex permittivity and permeability of lanthanum iron garnet filled PVDFpolymer composite using rectangular waveguide and Nicholson-Ross-Weir (NRW) method at X-band frequencies. Vol. 45, $\mathrm{N}^{\circ} 6$, pp. 1621-1625. Measurement ( 2012) 